\title{
Analysis of Six Bone-derived Factors in Plasma and Csf of Chinese Patients With Parkinson's Disease
}

\section{Xiang Chen}

Guangzhou Medical College First Affiliated Hospital

\section{Yuwan Lin}

Guangzhou Medical College First Affiliated Hospital

\section{Chaojun Chen}

Guangdong chinese medical integrated hospital

\section{Wenyuan Guo}

Guangzhou Medical College First Affiliated Hospital

\section{Miaomiao Zhou}

Guangzhou Medical College First Affiliated Hospital

Jiewen Qiu

Guangzhou Medical College First Affiliated Hospital

\section{Zhiling Zhang}

Guangzhou Medical College First Affiliated Hospital

\section{Mingshu Mo}

Guangzhou Medical College First Affiliated Hospital

\section{Liuyan Ding}

Guangzhou Medical College First Affiliated Hospital

\section{Panghai Ye}

Guangzhou Medical College First Affiliated Hospital

\section{Yijuan Wu}

Guangzhou Medical College First Affiliated Hospital

\section{Xiaoqin Zhu}

Guangzhou Medical University

\section{Yi Ren}

Florida State University Department of Biomedical Sciences

\section{Xingling Yang}

Xinjiang Medical University Affiliated First Hospital

\section{Xiaokang Zhang}

First Affiliated Hospital Of Gannan Medical College

\section{Zhuohua Wu}

Guangzhou Medical College First Affiliated Hospital

\section{Pingyi Xu ( $\nabla$ pingyixu@sina.com )}

First Affiliated Hospital of Guangzhou Medical University

\section{Short report}


Keywords: Parkinson's disease, bone-derived factors,Osteocalcin, Osteopontin囚C-reaction protein

Posted Date: June 24th, 2020

DOI: https://doi.org/10.21203/rs.3.rs-37163/v1

License: @ (i) This work is licensed under a Creative Commons Attribution 4.0 International License. Read Full License 


\section{Abstract}

\section{Background}

Parkinson's disease (PD) has a close relationship with osteoporosis and bone secretory proteins may be involved in disease progress.

\section{Objectives}

To detect the six bone-derived factors in plasma and CSFof patient swith PD and evaluate their correlations with CRP level, motor impairment and HY stage of the disease.

\section{Methods}

We included 250 PD patients and 250 controls. Levels of OCN, OPN,OPG, SO, BMP2 andDKK-1 in Plasma and CSF were measured by custom protein antibody arrays.Data were analyzed using Mann-Whitney U-testand Spearman's rank correlation.

\section{Results}

Plasma levels of OCN and OPN were correlated with CRP level and HY stage and motor impairment of PD.Furthermore, the plasma assessment with CSF detection may enhance their potential prediction on PD.

\section{Conclusions}

OCN and OPN may serve as potential biomarkers for PD. The inflammation response may be involved in the crosstalks between the two factors and PD.

\section{Introduction}

Parkinson's disease (PD) is one of the most common neurodegenerative disorders ${ }^{1}$. Patients with PD are at a high risk of osteoporotic fractures as a result of falls and reduced bone mass ${ }^{2}$. Osteoporosis may be a hidden non-motor syndrome of PD and fractures can also be found more commonly in the prodromal period of PD compared to controls ${ }^{3,4}$. This indicates that bone metabolism has a close relationship with the developing of PD ${ }^{4}$. Bone has traditionally been considered a structural organ that supports movement of the body and protects the internal organs. However, an increasing number of studies have shown that the skeleton could also be an active endocrine organ which secretes many kinds of bone-derived factors and contributes to pathophysiology of many diseases, such as Alzheimer's disease, diabetes mellitus, cardiovascular, chronic kidney disease ${ }^{5-8}$. Osteocalcin (OCN), Osteoprotegerin (OPG) and osteopontin (OPN) were reported upregulated in the plasma of PD patients ${ }^{9,10}$. However, we found that these case-control studies had relatively small sample sizes and the levels of these factors in cerebrospinal fluid (CSF) of PD patients remain unknown. In this study, we aimed to clarify the levels of OCN, OPN, OPG, Sclerostin (SO), Bone morphogenetic protein 2 (BMP2) and Dickkopf-1 (DKK-1) in the plasma and CSF of PD patients and identify candidate biomarkers for detection of PD. We also investigated the relevance of these bonederived factors with C-reaction protein (CRP) and motor dysfunction and disease progression.

\section{Methods}

1. Subjects 
This study enrolled 500 participants, including 250 with PD, and 250 healthy controls. All patients included in this study were tested negative for diabetes mellitus, thyroid disease or kidney disease. All participants were recruited from the First Affiliated Hospital of Guangzhou Medical University. This study was approved by the institutional Ethics Board Committee of the First Affiliated Hospital of Guangzhou Medical University and all participants provided written informed consent.

\section{Plasma and CSF}

Blood samples were collected from 200 healthy controls and 200 patients with PD and were centrifuged (2,500 g for 15 minutes) within 1 hour of collection. CSF samples were collected by lumbar puncture from 50 healthy controls and 50 patients with PD. CSF samples had no blood contamination (leukocyte number count fewer than $5 \mathrm{cells} / \mu \mathrm{L}$ and erythrocyte number fewer than 200 cells $/ \mu \mathrm{L}$ ). Sample aliquots were stored in cryotubes at $-80^{\circ} \mathrm{C}$ before testing . The levels of bone-derived factors were measured using custom protein antibody arrays (RayBiotech, www.raybiotech.com). CRP was measured using immunotubidometric assays.

\section{Statistical analysis}

Data statistics were carried out by SPSS, version 21 and GraphPad Prism version 6. Student's t tests or MannWhitney $U$ test was used to assess the difference of continuous variables between PD and control groups. Differences between groups for categorical variables were assessed using chi-square tests. Spearman coefficient calculation and Kruskal-Wallis $\mathrm{H}$ test were used to analyze possible correlations between parameters of interest. Receiver operating characteristics (ROC) curves were used to determine the diagnostic performance of studied bonederived factors in differentiating PD patients from controls. The accuracy of a biomarker in predicting PD was assessed by calculating the area under the ROC curve (AUC). Values of $P<0.05$ were considered significant.

\section{Results}

For this study, we obtained plasma samples from 200 PD patients and 200 age- and gender-matched controls, and CSF samples from 50 PD patients and 50 age- and gender-matched controls. More than $90 \%$ of plasma samples and $90 \%$ of CSF samples were detected in our statistical analyses. SO in CSF analysis was excluded due to low detection rates. In plasma, levels of OCN (11716.4(8052.7-14679.4) vs 4833.1(1953.1-8847.8), P<0.001) and OPN (16733.7 (12446.0-19981.3) vs 12333.7 (6341.4-16882.7), $\mathrm{P}<0.001)$ were increased in the PD patients relative to the controls. In contrast, levels of OPG (130.7(89.4-207) vs 169.5(113.1-245), P < 0.001) and BMP2 (11.8(8-20.9) vs 17.9(9.3731.5), $P<0.001$ ) were decreased in the PD patients compared to those in controls (Table 1, Fig. 1A-D). However, No significant difference in plasma SO and DKK1 levels were found between the two groups (Supplementary file). In CSF, patients with PD had significantly lower levels of OCN (14817.5(8145.9-18998.3) vs 18264(12835.5-22342.3), P = 0.002) (Fig. 1A) and OPG (204.3(107.3-307) vs 282.7(215.3-444.1), $\mathrm{P}=0.008$ ) (Fig. 1C) relative to healthy controls. There was no significant difference of OPN, BMP2 or DKK1 levels in CSF between the two groups (Table 1, Fig. 1B, D, and Supplementary file). 
Table 1

, Demographics and protein levels of six bone-derived factors in plasma and CSF in two groups

\begin{tabular}{|c|c|c|c|c|c|c|}
\hline \multirow{2}{*}{$\begin{array}{l}\text { Clinical } \\
\text { characteristics }\end{array}$} & \multicolumn{2}{|l|}{ Plasma } & \multirow{2}{*}{$\begin{array}{l}P \\
\text { value }\end{array}$} & \multicolumn{2}{|l|}{ CSF } & \multirow{2}{*}{$\begin{array}{l}P \\
\text { value }\end{array}$} \\
\hline & PD & Con & & PD & Con & \\
\hline $\begin{array}{l}\text { Gender } \\
\text { (Male/Female) }\end{array}$ & $113 / 87$ & $108 / 92$ & 0.688 & $29 / 21$ & $27 / 23$ & 0.84 \\
\hline Age (year) & $63.2(11.2)$ & 63.3(12.3) & 0.935 & $57.6(11.1)$ & $59(10.2)$ & 0.518 \\
\hline $\mathrm{H}-\mathrm{Y}$ & $2.3(0.8)$ & - & - & $2.2(0.6)$ & - & - \\
\hline UPDRS-III & 33.1(11.6) & - & - & $32.4(11.7)$ & - & - \\
\hline $\begin{array}{l}\text { CRP, median } \\
(\mathrm{IQR}), \mathrm{mg} / \mathrm{I}\end{array}$ & $3.3(1.8-4.5)$ & $2.1(1.1-2.9)$ & $\begin{array}{l}< \\
0.001\end{array}$ & $\begin{array}{l}0.15(0.12- \\
0.17)\end{array}$ & $\begin{array}{l}0.032(0.01- \\
0.04)\end{array}$ & 0.006 \\
\hline $\begin{array}{l}\text { OCN, median } \\
\text { (IQR), } \mathrm{pg} / \mathrm{ml}\end{array}$ & $\begin{array}{l}11716.4(8052.7- \\
14679.4)\end{array}$ & $\begin{array}{l}4833.1(1953.1- \\
8847.8)\end{array}$ & $\begin{array}{l}< \\
0.001\end{array}$ & $\begin{array}{l}\text { 14817.5(8145.9- } \\
18998.3)\end{array}$ & $\begin{array}{l}\text { 18264(12835.5- } \\
22342.3)\end{array}$ & 0.002 \\
\hline $\begin{array}{l}\text { OPN, median } \\
\text { (IQR), } \mathrm{pg} / \mathrm{ml}\end{array}$ & $\begin{array}{l}\text { 16733.7(12446.0- } \\
\text { 19981.3) }\end{array}$ & $\begin{array}{l}12333.7(6341.4- \\
16882.7)\end{array}$ & $\begin{array}{l}< \\
0.001\end{array}$ & $\begin{array}{l}9316.1(6955.1- \\
11378.3)\end{array}$ & $\begin{array}{l}8189.4(6629.2- \\
10736.8)\end{array}$ & 0.124 \\
\hline $\begin{array}{l}\text { OPG, median } \\
\text { (IQR), } \mathrm{pg} / \mathrm{ml}\end{array}$ & 130.7(89.4-207) & $\begin{array}{l}\text { 169.5(113.1- } \\
245)\end{array}$ & $<$ & $\begin{array}{l}204.3(107.3- \\
307)\end{array}$ & $\begin{array}{l}282.7(215.3- \\
444.1)\end{array}$ & 0.008 \\
\hline $\begin{array}{l}\text { SO, median } \\
(\mathrm{IQR}), \mathrm{pg} / \mathrm{ml}\end{array}$ & $\begin{array}{l}\text { 651.4(321.1- } \\
1068.7)\end{array}$ & $\begin{array}{l}748.6(372.6- \\
1272.2)\end{array}$ & 0.147 & - & - & - \\
\hline $\begin{array}{l}\text { BMP2, median } \\
\text { (IQR), } \mathrm{pg} / \mathrm{ml}\end{array}$ & 11.8(8-20.9) & $17.9(9.37-31.5)$ & $\begin{array}{l}< \\
0.001\end{array}$ & 44.3(22-84.1) & $45(27.5-60.5)$ & 0.900 \\
\hline $\begin{array}{l}\text { DKK1, median } \\
\text { (IQR), } \mathrm{pg} / \mathrm{ml}\end{array}$ & 45.6(15.8-141.1) & $\begin{array}{l}63.6(24.8- \\
135.4)\end{array}$ & 0.067 & $16.2(5.2-56.3)$ & $\begin{array}{l}33.46(18.1- \\
54.7)\end{array}$ & 0.080 \\
\hline
\end{tabular}

To evaluate whether these bone-derived factors could be the potential biomarkers for PD risk, the natural-logarithm values of these levels were analyzed using ROC curves. Compared with the NC group, the AUCs for the plasma and CSF levels of OCN in PD patients were $0.863(95 \% \mathrm{Cl}=0.771-0.928)$ and $0.67(95 \% \mathrm{Cl}=0.559-0.769)$, respectively. Moreover, the AUC was higher when combined assessment of plasma and CSF OCN in PD patients, at $0.869(95 \% \mathrm{Cl}$ $=0.778-0.993)$ (Fig. 1E). The AUCs for plasma, CSF, and the combined assessment of OPN when comparing PD patients with control groups were $0.73(95 \% \mathrm{Cl}=0.632-0.814), 0.589(95 \% \mathrm{Cl}=0.486-0.687)$, and $0.739(95 \% \mathrm{Cl}=$ $0.641-0.822$ ), respectively (Fig. 1F). The AUCs for plasma, CSF, and the combined assessment of OPG and BMP2 were less than 0.7 (Supplementary file). Further, the plasma level of OCN and OPN were correlated with the Hoehn and Yahr disease stage (Fig. 1H, K) and the Movement Disorders Society-Unified Parkinson's Disease Rating Scale (MDS-UPDRS)-III score (UPDRS III) (Fig. 1. G, J). Inflammation was involved both in the pathology of osteoporosis and PD, we next analyzed the relationship between CRP and these bone-derived factors. We found that significant correlations existed between plasma OCN and OPN and CRP levels in PD (Fig. 1I, L). However, no correlation was identified between these factors and CRP in CSF.

Table 1, Demographics and protein levels of six bone-derived factors in plasma and CSF in two groups.

Data are represented as Mean (SD), median (IQR) or $n$. P value was considered significant when $<0.05$, PD Parkinson's disease, Con Controls, H-YHoehn and Yahr, UPDRS-III Unified Parkinson's Disease Rating Scale part 
III(motor score). CRP C-reaction protein,OCNOsteocalcin,OPN Osteopontin,OPGOsteoprotegerin, SO Sclerostin, BMP2 Bone morphogenetic protein 2, DKK-1Dickkopf-1, IQR Interquartile range.

\section{Discussion}

Although a number of studies have focused on the question of comorbidity of PD and osteoporosis. The relationship between bone derived factors and risk of PD still remain unclear. In the present study, we tested six bone-derived factors and found increased levels of OCN and OPN and decreased levels of OPG and BMP2 in plasma of PD patients. And levels of OCN and OPG were lower in CSF of PD relative to controls. Furthermore, we identified that plasma OCN and OPN was correlated with the disease stage and motor impairment. CRP was correlated with plasma levels of OCN and OPN. Combined assessment of plasma and CSF of OCN or OPN was a better biomarker for differentiating PD patients from healthy controls.

$\mathrm{OCN}$ is one of the most abundant bone-specific non-collagenous protein secreted primarily by osteoblasts and is often used as a biomarker for bone formation. In recent years, OCN has been regarded as a bone-derived hormone that plays important roles in physiological and pathological processes ${ }^{11}$. In peripheral, OCN acts as a regulator of the activity of osteoclasts and also maintains the energy homeostasis ${ }^{12}$. In brain, the uncarboxylated form of OCN can accumulate in the brainstem, thalamus and hypothalamus, and influencing various neurotransmitters synthesis and signalings ${ }^{13}$. In PD, OCN could correct motor dysfunction and reduce dopaminergic neuronal injury via the AKT/GSK3 $\beta$ signaling pathway in an animal model of $\mathrm{PD}^{14}$. In this study, we found that the plasma level of OCN was increased, however the CSF level of OCN was decreased in PD patients. This may be accounted for the different roles of OCN in peripheral circulation and central nervous system. Given the role of OCN in protecting dopaminergic neurons, improving cognition and preventing anxiety and depression,low expression level of OCN in CSF indicates its involvement in motor and non-motor symptoms of PD. The role of OCN in the pathophysiology of PD is obscure. Plasma level of OCN correlated with CRP and $\mathrm{H}-\mathrm{Y}$ stage of PD patients indicates inflammation may be a potential bridge between OCN and progression of PD. However, no correlation was identified between OCN and CRP in CSF, indicating that other mechanism are involved in PD in central nervous system. Furthermore, compared to analyzing plasma or CSF OCN alone, the combined assessment is more effective in differentiating PD patients from healthy controls and plasma level of OCN was correlated with the disease stage and motor impairment. All these results highlight the important roles of OCN in the pathology of PD.

OPN is a glycosylated phosphoprotein belonging to the small integrin binding ligand, N-linked glycoprotein (SIBLING) family of proteins ${ }^{15}$. It is highly expressed by bone marrow-derived myelomonocytic cells and can act both as a matrix protein and as a cytokine. As a multifunctional protein, OPN plays significant roles in regulating reactive oxygen species production, levels of inflammatory cytokines and apoptotic signals ${ }^{7}$. OPN was reported to involve in the pathology of various brain diseases, such as Alzheimer's disease (AD), multiple sclerosis, and traumatic brain injury via neuroprotective and repair-promoting effects ${ }^{7,16}$. OPN is also expressed in substantia nigra and in nigral dopaminergic neurons and its expression is decreased in surviving dopaminergic neurons in PD, suggesting a potential role of OPN in neuroprotection of PD. However, OPN knockout mice displayed less nigral cell death and a decreased glial response in a 1-methyl-4-phenyl-1, 2, 3, 6-tetrahydropyridine (MPTP) induced animal model of PD ${ }^{16,}$

17. This suggests that OPN may act as a double-edged sword triggering neuronal toxicity or functioning as a neuroprotectant in PD. Inflammatory response may be one of the mechanism of OPN in PD as there is significant correlation between OPN and CRP. Walter Maetzler have reported that OPN was upregulated both in the plasma and CSF of PD patients ${ }^{17}$, however we only identified higher level of $O C N$ in the plasma of PD patients. The incongruence 
may be due to difference in disease stage, ethnic or environmental factors and more studies are needed to reveal the function of OPN in PD.

In conclusion, we analyzed six bone-derived factors and revealed abnormal expression levels of OCN, OPN, OPG and BMP2 in plasma or CSF of PD. We identified that plasma level of OCN and OPN were correlated with CRP, H-Y stage and motor impairment. Our study also suggests that combined assessment of plasma and CSF of OCN or OPN may enhance their potential for predicting PD. Inflammation response may be involved in the cross-talks between these two factors and PD. These findings may contribute to the functional understanding of PD pathophysiology. However, further studies are needed to confirm our findings and to illustrate the roles of inflammation or immune mechanisms involved in the factors on PD.

\section{Abbreviations}

PD

Parkinson's disease

CSF

Cerebrospinal fluid

CRP

C-reaction protein

$\mathrm{H}-\mathrm{Y}$ stage

Hoehn and Yahr disease stage

OCN

Osteocalcin

OPN

Osteopontin

OPG

Osteoprotegerin

so

Sclerostin

BMP2

Bone morphogenetic protein 2

DKK-1

Dickkopf-1

ROC

Receiver operating characteristics

AUC

Area under the ROC curve

MDS-UPDRS-III score (UPDRS)

III Movement Disorders Society-Unified Parkinson's Disease Rating Scale

SIBLING

Small integrin binding ligand, $\mathrm{N}$-linked glycoprotein

AD

Alzheimer's disease

MPTP

1-methyl-4-phenyl-1, 2, 3, 6-tetrahydropyridine 


\section{Declarations}

\section{Ethics approval and consent to participate}

This study was approved by the Medical Ethics Committee of The First Affiliated Hospital of Guangzhou Medical University.(Guangzhou, China), and the study participants provided written informed consent.

\section{Consent for publication}

Not applicable

\section{Availability of data and materials}

The datasets analyzed during the current study are available from the corresponding author on reasonable request.

\section{Competing interests}

The authors declare that they have no competing interests.

\section{Funding}

National Key R\&D Program of China(2016YFC130660ه2017YFC1306002), National Natural Science Foundation of China (81901282, 81870992『81870856هU1603281, 81771891, 81401462), Guangdong Basic and Applied Basic Research Foundation (2019A1515011189,2020A1515010986,2020A1515010985), Science and Technology Planning Project of Guangdong Province (A2018315),Technology project of Guangzhou (2018-1202-SF-0019).

\section{Authors' contributions}

1) Research project: A. Conception:PingyiXu,ZhuohuaWu,B. Organization:Xiang Chen,Mingshu Mo,Xingling Yang,C. Execution:Yuwan Lin, Chaojun Chen, Miaomiao Zhou,JiewenQiu,Liuyan Ding,Panghai Ye;

2) Statistical Analysis: A, B. Execution:WenyuanGuo,Yijuan Wu,Zhiling Zhang,MingshuMo囚C. Review and Critique:ZhuohuaWu;Yi Ren,Xiaoqin Zhu

3) Manuscript: A. Writing of the first draft:Xiang Chen, B: Review and Critique: Xiaokang Zhang, ZhuohuaWu, PingyiXu;

\section{Acknowledgements}

Not applicable

\section{References}

1. Armstrong MJ, Okun MS. Diagnosis and Treatment of Parkinson Disease: A Review. JAMA. 2020;323(6):54860.

2. Torsney KM, Noyce AJ, Doherty KM, Bestwick JP, Dobson R, Lees AJ. Bone health in Parkinson's disease: a systematic review and meta-analysis. J Neurol Neurosurg Psychiatry. 2014;85(10):1159-66.

3. Camacho-Soto A, Gross A, Searles Nielsen S, et al. Fractures in the prodromal period of Parkinson disease. Neurology 2020. 
4. Metta V, Sanchez TC, Padmakumar C. Osteoporosis. A Hidden Nonmotor Face of Parkinson's Disease. Int Rev Neurobiol. 2017;134:877-90.

5. Rentsendorj A, Sheyn J, Fuchs D-T, et al. A novel role for osteopontin in macrophage-mediated amyloid- $\beta$ clearance in Alzheimer's models. Brain Behav Immun. 2018;67:163-80.

6. Vervloet MG, Massy ZA, Brandenburg VM, et al. Bone: a new endocrine organ at the heart of chronic kidney disease and mineral and bone disorders. Lancet Diabetes Endocrinol. 2014;2(5):427-36.

7. Rentsendorj A, Sheyn J, Fuchs DT, et al. A novel role for osteopontin in macrophage-mediated amyloid-beta clearance in Alzheimer's models. Brain Behav Immun. 2018;67:163-80.

8. Xu Y, Ma X, Pan X, He X, Xiao Y, Bao Y. Correlations between serum concentration of three bone-derived factors and obesity and visceral fat accumulation in a cohort of middle aged men and women. Cardiovasc Diabetol. 2018;17(1):143.

9. Sato Y, Kaji M, Tsuru T, Satoh K, Kondo I. Vitamin K deficiency and osteopenia in vitamin D-deficient elderly women with Parkinson's disease. Arch Phys Med Rehabil. 2002;83(1):86-91.

10. Alrafiah A, Al-Ofi E, Obaid MT, Alsomali N. Assessment of the Levels of Level of Biomarkers of Bone Matrix Glycoproteins and Inflammatory Cytokines from Saudi Parkinson Patients. BioMed research international 2019;2019:2690205.

11. Wei J, Karsenty G. An overview of the metabolic functions of osteocalcin. Rev Endocr Metab Disord. 2015;16(2):93-8.

12. Mizokami A, Kawakubo-Yasukochi T, Hirata M. Osteocalcin and its endocrine functions. Biochem Pharmacol. 2017;132:1-8.

13. Shan C, Ghosh A, Guo XZ, et al. Roles for osteocalcin in brain signalling: implications in cognition- and motorrelated disorders. Mol Brain. 2019;12(1):23.

14. Guo XZ, Shan C, Hou YF, et al. Osteocalcin Ameliorates Motor Dysfunction in a 6-Hydroxydopamine-Induced Parkinson's Disease Rat Model Through AKT/GSK3beta Signaling. Front Mol Neurosci. 2018;11:343.

15. Pang X, Gong K, Zhang X, Wu S, Cui Y, Qian B-Z. Osteopontin as a multifaceted driver of bone metastasis and drug resistance. Pharmacological research. 2019;144:235-44.

16. Carecchio M, Comi C. The role of osteopontin in neurodegenerative diseases. J Alzheimers Dis. 2011;25(2):17985.

17. Maetzler W, Berg D, Schalamberidze N, et al. Osteopontin is elevated in Parkinson's disease and its absence leads to reduced neurodegeneration in the MPTP model. Neurobiol Dis. 2007;25(3):473-82.

\section{Figures}



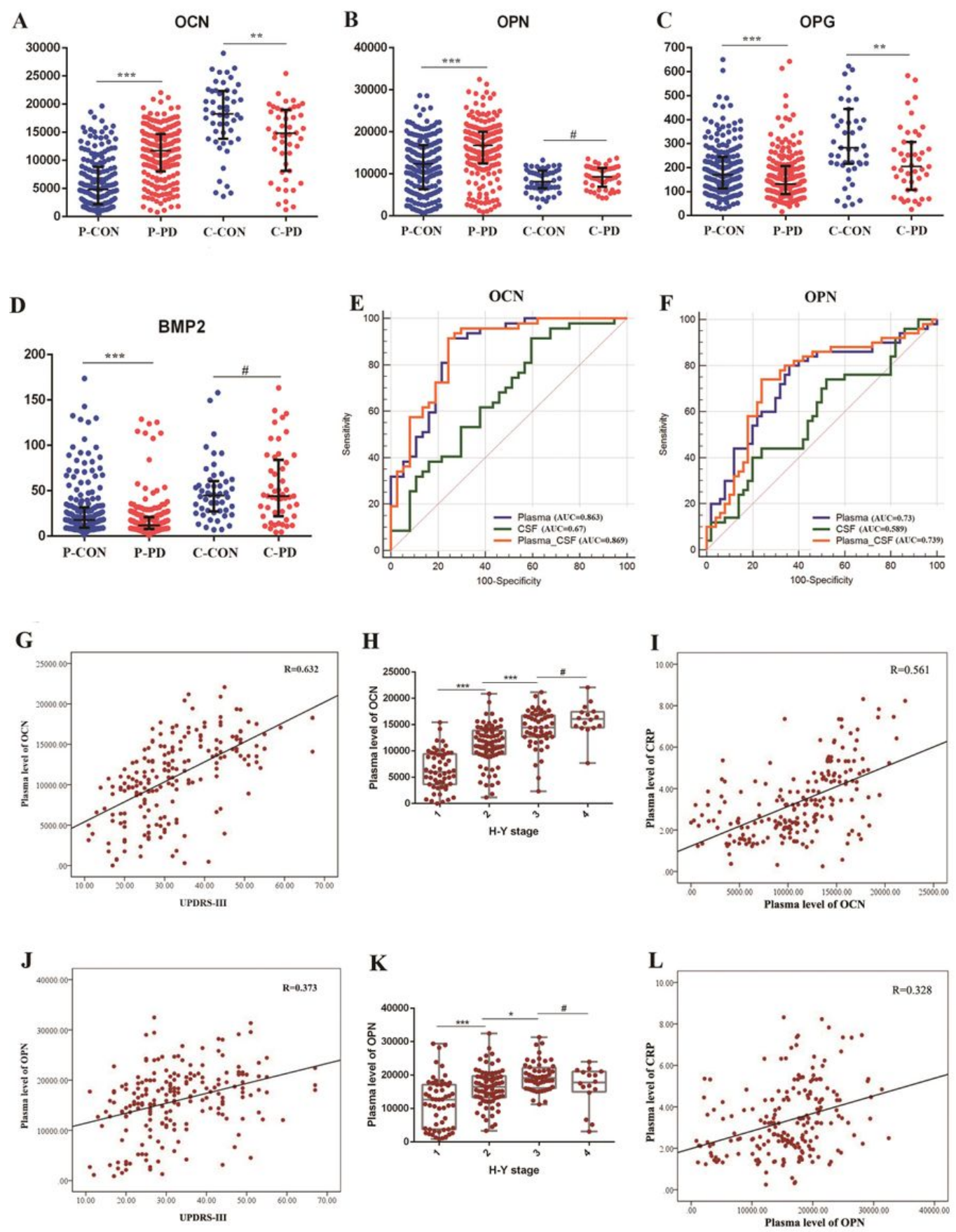

Figure 1

Expression levels, ROCanalysis and correlationanalysis of selected bone-derived factors of PD relative to healthy controls. A-D present the concentrations of OCN, OPN,OPGand BMP2 1 in plasma (P) and cerebrospinalfluid (C) of Parkinson's disease (PD) and healthy controls (CON). Data are presented as median and IQR $(* \star P<0.01, \star \star \star P<<0.001$, $\# P>0.05$ from Mann-Whitney test). E-F,Receiver operating characteristics curves of plasma, CSF andcombined of plasma and CSF (Plasma-CSF) ofOCNand OPN were analyzed.AUC, area under the curve.G, Scatter diagram of the correlation between the plasma level of OCN and UPDRS-IIlof PD patients $(r=0.632, P<0.001) . H$, The relationship of

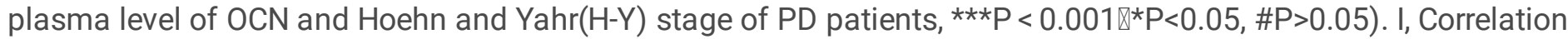
between the plasma level of OCN and C-reactionprotein (CRP) of PD patients $(r=0.561, P<0.001)$. J, Scatter diagram of the correlation between the plasma level of OPN and UPDRS-IIlof PD patients( $r=0.373, P<0.001)$. K, The 
relationship of plasma level of OPN and H-Y stage of PD patients, $* \star * P<0.001 \rrbracket * P<0.05, \# P>0.05)$. I, Correlation between the plasma level of OPN and C-reactionprotein (CRP) of PD patients $(r=0.328, P<0.001)$.

\section{Supplementary Files}

This is a list of supplementary files associated with this preprint. Click to download.

- Supplementaryfile.docx 